\title{
PROPRIEDADES TERMOFÍSICAS DE SOLUÇÕES MODELO SIMILARES A CREME DE LEITE ${ }^{1}$
}

\author{
Silvia Cristina Sobottka Rolim de MOURA' ${ }^{2, *}$, Alfredo de Almeida VITALI², Vitor Correia Lima FRANÇA ${ }^{3}$
}

\begin{abstract}
RESUMO
A demanda de creme de leite UHT tem aumentado significativamente. Diversas empresas diversificaram e aumentaram sua produção, visto que o consumidor, cada vez mais exigente, almeja cremes com ampla faixa de teor de gordura. O objetivo do presente trabalho foi determinar a densidade, viscosidade aparente e difusividade térmica, de soluções modelo similares a creme de leite, na faixa de temperatura de 30 a $70^{\circ} \mathrm{C}$, estudando a influência do teor de gordura e da temperatura nas propriedades físicas dos produtos. O delineamento estatístico aplicado foi o planejamento $3 \times 5$, usando níveis de teor de gordura e temperatura fixos em $15 \%, 25 \%$ e $35 \% ; 30^{\circ} \mathrm{C}, 40^{\circ} \mathrm{C}, 50^{\circ} \mathrm{C}, 60^{\circ} \mathrm{C}$ e $70^{\circ} \mathrm{C}$, respectivamente (STATISTICA 6.0). Manteve-se constante a quantidade de carboidrato e de proteína, ambos em $3 \%$. A densidade foi determinada pelo método de deslocamento de fluidos em picnômetro; a difusividade térmica com base no método de Dickerson e a viscosidade aparente foi determinada em reômetro Rheotest 2.1. Os resultados de cada propriedade foram analisados através de método de superfície de resposta. No caso destas propriedades, os dados obtidos apresentaram resultados significativos, indicando que o modelo representou de forma confiável a variação destas propriedades com a variação da gordura $(\%)$ e da temperatura $\left({ }^{\circ} \mathrm{C}\right)$.
\end{abstract}

Palavras-chave: difusividade térmica; viscosidade; densidade; soluções modelo; creme de leite.

\section{SUMMARY}

THERMOPHYSICAL PROPERTIES OF MODEL SOLUTIONS SIMILAR TO CREAM. The requirement of UHT cream has been increased considerably. Several industries varied and increased their production, since consumers, more and more exigent, are demanding creams with a wide range of fat content. The objective of the present research was to determine the density, viscosity and thermal diffusivity of model solutions similar to cream. The range of temperature varied from $30^{\circ} \mathrm{C}$ to $70^{\circ} \mathrm{C}$ in order to study the influence of fat content and temperature in the physical properties of cream. The statistic method applied was the factorial $3 \times 5$ planning, with levels of fat content and temperature fixed in $15 \%, 25 \%$ and $35 \% ; 30^{\circ} \mathrm{C}, 40^{\circ} \mathrm{C}, 50^{\circ} \mathrm{C}, 60^{\circ} \mathrm{C}$ and $70^{\circ} \mathrm{C}$, respectively (STATISTICA 6.0 ). Both carbohydrates and proteins content were fixed at $3 \%$. The density was determined by a method based on the displacement of fluids in a picnometer; the thermal diffusivity by Dickerson method and the viscosity by Rheotest 2.1 rheometer. The results of each property were analysed by the response surface method. For all the properties the results were significant, indicating that this model represented considerably the changes of thermal and physical properties of cream according to fat content and temperature variations.

Keywords: thermal diffusivity; viscosity; density; model solutions; cream.

\footnotetext{
${ }_{1}^{1}$ Recebido para publicação em 25/01/00. Aceito para publicação em 29/06/01.

${ }^{2}$ Centro de Tecnologia de Hortifrutícolas - FRUTHOTEC/Instituto de Tecnologia de Alimentos - ITAL, Avenida Brasil, 2880, Campinas - SP, 13073-001, Brasil, email: smoura@ital.org.br

${ }^{3}$ Bolsista de Iniciação Científica CNPq - PIBIC

${ }^{*}$ A quem a correspondência deve ser enviada.
}

\section{1 - INTRODUÇÃO}

Com a demanda de creme de leite UHT crescente, diversas empresas diversificaram e aumentaram sua produção para agradar ao consumidor que almeja cremes com maior variedade de faixas de teor de gordura. $\mathrm{O}$ creme com baixo teor de gordura (12-18\%) pode ser adicionado ao café e o com alto teor de gordura (35-48\%) pode ser utilizado no preparo de doces e sobremesas [8].

O dimensionamento dos equipamentos utilizados no processamento de creme de leite UHT, principalmente os trocadores de calor e outros que requerem bombeamento do produto, exigem dados precisos das propriedades térmicas dos produtos (condutividade e difusividade térmicas e calor específico) e de como essas propriedades se comportam durante o processo, em função da temperatura.

PHIPPS [10] desenvolveu equações para creme de leite que mostraram a relação entre a viscosidade, o teor de gordura (até $50 \%$ ) e a temperatura $\left(40-80^{\circ} \mathrm{C}\right.$ ). O mesmo foi feito pelo autor para a densidade.

Algumas propriedades termofísicas de um sistema composto por duas fases, como o creme de leite, tais como a densidade e o calor específico podem ser medidas com facilidade, enquanto a viscosidade e a condutividade térmica, até então, não podiam ser preditas com precisão [4]. A necessidade de se conhecer as propriedades térmicas com precisão tem levado ao estudo da influência da composição e da temperatura nestas propriedades.

Em 1980, POLLEY et al [11], publicaram uma coleção de tabelas de propriedades térmicas em diversos alimentos, entre eles carnes, frutas, legumes, leite e cereais. São encontrados nestas tabelas alguns dados de calor específico para cremes porém, incompletos.

Já em 1986, OKOS [9] compilou equações que predizem várias propriedades termofísicas de uma diversa gama de alimentos, tais como leite, sucos, óleos, derivados do leite, especialmente o creme. Estas equações correlacionam as propriedades termofísicas com a temperatura e a composição centesimal do alimento a ser analisado.

Em um trabalho recente, HINRICHS [5] estudou as propriedades reológicas de leite e de creme, verificando a influência do teor de gordura e da temperatura na viscosidade destes produtos. Verificou-se, também, os danos causados quando o creme de leite estava submetido a bombeamento ou agitação, principalmente quando continha mais que $40 \%$ de gordura.

O creme de leite é uma emulsão de gordura em leite, produto de composição muito similar a do leite 
integral, exceto pela alta quantidade de gordura de leite que é adicionada para caracterizar o tipo de creme a ser produzido. A modelagem de creme de leite com gordura de leite foi proposta e executada com sucesso por FERNÁNDEZ-MARTÍN \& MONTES [4], entretanto, uma mistura de leite desnatado e óleo vegetal também obteve bons resultados em trabalhos mais recentes como TEPPER et al [13] e TEPPER \& KUANG [12].

Desta forma, para este estudo, o creme de leite foi considerado uma emulsão bifásica que consiste em uma fase contínua de água e uma fase dispersa de gordura vegetal, mantendo-se fixos os teores de carboidrato e de proteína, ambos em $3 \%$. Obviamente que esse sistema é não newtoniano, devido a eventuais alterações do tamanho das partículas que compõem a fase dispersa, em função da taxa de cisalhamento. Entretanto, em estudos de engenharia, é comum se estudar a reologia do produto determinando-se sua viscosidade aparente.

O objetivo do presente trabalho foi determinar experimentalmente a densidade, a viscosidade aparente e a difusividade térmica, de soluções modelo similares a creme de leite, na faixa de temperatura de 30 a $70^{\circ} \mathrm{C}$, estudando a influência do teor de gordura e da temperatura nas propriedades físicas dos produtos.

\section{2 - MATERIAIS E MÉTODOS}

\section{1 - Materiais}

As soluções modelo foram preparadas seguindo uma distribuição de componentes dada por um tratamento estatístico [2], tentando-se simular um creme de leite. Foram varridos os teores de gordura (15\% a $35 \%)$ e as temperaturas $\left(30^{\circ} \mathrm{C}, 40^{\circ} \mathrm{C}, 50^{\circ} \mathrm{C}, 60^{\circ} \mathrm{C}\right.$ e $\left.70^{\circ} \mathrm{C}\right)$, mantendo-se fixa a quantidade de carboidrato e de proteína, ambas em 3,0\%. Para estabilizar a emulsão, utilizou-se goma carragena a $0,5 \%$. Os teores foram definidos por testes preliminares.

\subsection{1 - Preparação das soluções}

Inicialmente preparou-se uma solução $2,5 \%$ de caseína comercial em água, ajustou-se o pH até 10,5 com NaOH $1,0 \mathrm{~N}$, e aqueceu-se por 5 minutos a $90^{\circ} \mathrm{C}$. Nestas condições a caseína apresenta sua melhor capacidade emulsificante [6]. Depois o $\mathrm{pH}$ foi ajustado até um valor neutro. Uma vez pronta a solução de caseína, elevou-se a temperatura até $70^{\circ} \mathrm{C}$ e promoveu-se agitação para melhor solubilização da carragena [7]. Logo após adicionou-se o carboidrato e a gordura, também sob agitação constante.

\section{2 - Métodos}

\subsection{1 - Densidade $(\rho)$}

Determinada nas temperaturas de 30, 40, 50, 60 e $70^{\circ} \mathrm{C}$ pelo método de deslocamento de fluidos em picnômetro, segundo Norma n. ${ }^{\circ} 985.19$ da AOAC [14]. Os picnômetros foram calibrados (água destilada) e os dados coletados em triplicata.

\subsection{2 - Difusividade térmica $(\alpha)$}

O método usado na determinação da difusividade térmica foi baseado no método de DICKERSON [3], empregando-se o seguinte aparato: uma célula, formada por um tubo de aço inox, com $5,0 \mathrm{~cm}$ de diâmetro e $29,8 \mathrm{~cm}$ de altura, com dois termopares acoplados, devidamente calibrados - um na superfície, para tomadas de medidas de temperatura nesta posição, outro, tipo agulha, disposto no plano central da amostra (Figura 1). Nessa determinação, faz-se necessária a eliminação de correntes convectivas no interior da célula. Para tanto utiliza-se pectina BTM, a $5 \%$, combinada com $\mathrm{CaCl}_{2}$, a $4 \%$ do total de pectina, na formulação da amostra, levando à gelificação do produto, sendo esta uma técnica eficiente mesmo com elevação de temperatura. Considera-se que a forma gel não altera substancialmente a determinação da propriedade difusividade.

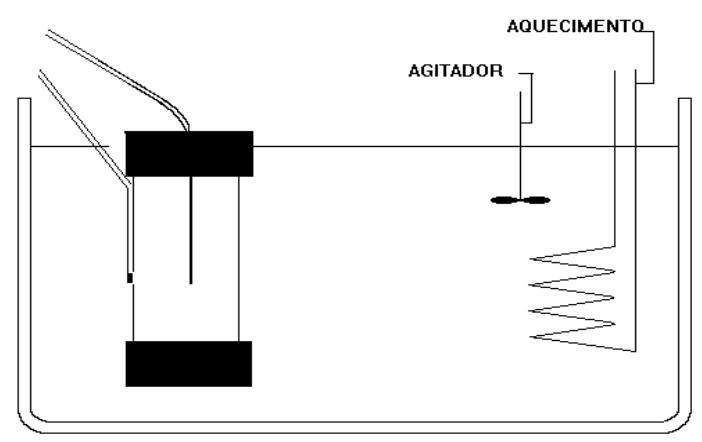

FIGURA 1. Esquema do aparato para medição de difusividade térmica.

O aparato de medição é então disposto em banho térmico de água e etilenoglicol, a $0^{\circ} \mathrm{C}$. Após o equilíbrio térmico entre a célula e o banho, inicia-se o aquecimento, a uma taxa de $0,7^{\circ} \mathrm{C} / \mathrm{min}$. O aquecimento será interrompido quando a temperatura interna da amostra atingir aproximadamente $85^{\circ} \mathrm{C}$. As medidas de temperatura foram tomadas em intervalos de 1 minuto, por meio de uma placa aquisitora de dados, modelo DAS-TC. Os dados obtidos serviram de base para o tratamento gráfico dos perfis de evolução das temperaturas externa e interna, além de permitirem uma monitoração da taxa de elevação de temperatura do banho térmico. A difusividade térmica foi calculada pela seguinte equação:

$\alpha=A \cdot R^{2 / 4} \cdot\left(T_{\text {ext }}-T_{\text {int }}\right)$

onde:

$\alpha=$ difusividade térmica

$\mathrm{A}=$ taxa de subida de temperatura do banho $\left({ }^{\circ} \mathrm{C} / \mathrm{min}\right)$

$R=$ raio da célula $(m)$

$($ Text - Tint $)=$ diferença entre $a$ temperatura externa e a temperatura interna da célula $\left({ }^{\circ} \mathrm{C}\right)$ 
O valor de A assumido para o cálculo é o valor obtido a partir da fase constante de subida da temperatura do banho. O valor da diferença de temperatura externa e interna diminui à medida que a temperatura do banho aumenta; portanto, o valor calculado para a difusividade é obtido para cada temperatura, fazendo-se posteriormente a média dos valores na faixa estudada.

\subsection{3 - Viscosidade aparente $\left(\mu_{\mathrm{ap}}\right)$}

Determinada através do reômetro Rheotest 2.1 (VEBMLW Alemanha), nas temperaturas de 30, 40 50, 60 e $70^{\circ} \mathrm{C}$.

O creme de leite é considerado um fluido tixotrópico, obedecendo a equação da lei de potência seguinte:

$\tau=\kappa \cdot \gamma^{n}$

onde

$\tau=$ tensão de cisalhamento $(\mathrm{Pa})$;

$\gamma=$ velocidade de deformação $\left(\mathrm{s}^{-1}\right)$;

$\kappa=0$ coeficiente de consistência do fluido (dina. $\mathrm{s}^{n} / \mathrm{cm}^{2}$ );

$\mathrm{n}=\mathrm{o}$ índice de comportamento do fluido.

Define-se a viscosidade aparente como sendo

$$
\mu_{\mathrm{ap}}=\frac{\tau}{\gamma}
$$

Rerranjando-se as equações (III) e (IV) tem-se:

$\mu_{\mathrm{ap}}=\mathrm{K} \cdot \gamma^{\mathrm{n}}$

Através da regressão linear de Log $\tau$ versus Log $N$, onde $\mathrm{N}$ é a velocidade do cilindro do reômetro (rpm), tira-se o valor de $n$, que é o coeficiente angular da curva. O coeficiente de consistência do fluido $(k)$ é obtido graficamente plotando-se a curva Log $\tau$ versus $\log \gamma$. A tensão de cisalhamento $(\tau)$, velocidade de deformação $(\gamma)$ e viscosidade aparente $\left(\mu_{\mathrm{ap}}\right)$, são fornecidos pelo reômetro, a cada ensaio.

Para obtenção adequada do diagrama de tensão de cisalhamento versus velocidade de deformação, os ensaios começam da menor velocidade de rotação (rpm) até se atingir o máximo de velocidade permitido pelo reômetro. Depois, diminui-se gradativamente a velocidade.

\subsection{4 - Tratamento estatístico}

O delineamento estatístico aplicado foi o planejamento $3 \times 5$ ! [2], usando-se níveis de teor de gordura e temperatura fixos entre $15 \%$ e $35 \%$ e $30^{\circ} \mathrm{C}$, e $70^{\circ} \mathrm{C}$, respectivamente, mantendo-se fixas as concentrações de carboidratos e proteínas, ambas a 3,0\% (Tabela 1). Utilizou-se o programa STATISTICA 6.0 para análise dos efeitos principais e das interações entre os tratamentos, fornecendo uma superfície de resposta [1] da propriedade de interesse em função do teor de gordura e da temperatura. Para cada propriedade física foram realizados 17 ensaios, seguindo-se o plane- jamento inicial, para a viabilização da superfície de resposta.

De acordo com a metodologia proposta por BOX et al [2], as variáveis gordura e temperatura foram codificadas de acordo com as equações abaixo:

$\mathrm{X}_{1}=\%$ gordura -25
10

$\mathrm{X}_{2}=$ Temperatura -50

As superfícies obtidas pelo programa STATISTICA seguem o modelo estatístico quadrático:

$\mathrm{Y}=\mathrm{B} 0+\mathrm{B} 1{ }^{*} \mathrm{X} 1+\mathrm{B} 2^{*} \mathrm{X} 1^{2}+\mathrm{B} 3^{*} \mathrm{X} 2+\mathrm{B} 4^{*} \mathrm{X} 2^{2}+\mathrm{B} 5^{*} \mathrm{X} 1^{*} \mathrm{X} 2$

Onde $\mathrm{Y}$ pode ser a densidade, difusividade ou viscosidade aparente; B0 é a identidade e B1 a B5 representam os efeitos principais e efeitos de interação entre as variáveis $\mathrm{X} 1$ e $\mathrm{X} 2$.

TABELA 1. Composição dos ensaios utilizada para determinar as propriedades físicas.

\begin{tabular}{|c|c|c|c|c|c|c|c|}
\hline Ensaio & $\begin{array}{c}\text { Gordura } \\
(\%)\end{array}$ & $\begin{array}{c}\text { Temperatura } \\
\left({ }^{\circ} \mathrm{C}\right)\end{array}$ & $X_{1}$ & $\mathrm{X}_{2}$ & $\begin{array}{c}\text { Caseína } \\
(\%)\end{array}$ & $\begin{array}{c}\text { Carboidrato } \\
(\%)\end{array}$ & $\begin{array}{l}\begin{array}{c}\text { Água } \\
(\%)\end{array}\end{array}$ \\
\hline 1 & 15 & 30 & -1 & -2 & 3,0 & 3,0 & 79 \\
\hline 2 & 15 & 40 & -1 & -1 & 3,0 & 3,0 & 79 \\
\hline 3 & 15 & 50 & -1 & 0 & 3,0 & 3,0 & 79 \\
\hline 4 & 15 & 60 & -1 & 1 & 3,0 & 3,0 & 79 \\
\hline 5 & 15 & 70 & -1 & 2 & 3,0 & 3,0 & 79 \\
\hline 6 & 25 & 30 & 0 & -2 & 3,0 & 3,0 & 69 \\
\hline 7 & 25 & 40 & 0 & -1 & 3,0 & 3,0 & 69 \\
\hline 8 & 25 & 50 & 0 & 0 & 3,0 & 3,0 & 69 \\
\hline 9 & 25 & 50 & 0 & 0 & 3,0 & 3,0 & 69 \\
\hline 10 & 25 & 50 & 0 & 0 & 3,0 & 3,0 & 69 \\
\hline 11 & 25 & 60 & 0 & 1 & 3,0 & 3,0 & 69 \\
\hline 12 & 25 & 70 & 0 & 2 & 3,0 & 3,0 & 69 \\
\hline 13 & 35 & 30 & 1 & -2 & 3,0 & 3,0 & 59 \\
\hline 14 & 35 & 40 & 1 & -1 & 3,0 & 3,0 & 59 \\
\hline 15 & 35 & 50 & 1 & 0 & 3,0 & 3,0 & 59 \\
\hline 16 & 35 & 60 & 1 & 1 & 3,0 & 3,0 & 59 \\
\hline 17 & 35 & 70 & 1 & 2 & 3,0 & 3,0 & 59 \\
\hline
\end{tabular}

\section{3 - RESULTADOS E DISCUSSÃO}

Os resultados de densidade, viscosidade aparente e difusividade estão dispostos na Tabela 2.

Os dados da Tabela 2 possibilitaram construir os gráficos de superfície de resposta para as propriedades termofísicas: densidade; viscosidade aparente; e difusividade térmica, em função do teor de gordura e da temperatura.

\section{1 - Densidade $(\rho)$}

De acordo com a Figura 2, a densidade aumentou com a elevação da porcentagem de gordura, e diminuiu com o acréscimo da temperatura. O modelo de segunda ordem obtido para densidade (DENS) está apresentado na própria Figura. 
TABELA 2: Resultados das propriedades: densidade, difusividade térmica e viscosidade aparente a $54 \mathrm{rpm}$.

\begin{tabular}{|c|c|c|c|}
\hline Ensaio & $\rho\left(\mathrm{kg} / \mathrm{m}^{3}\right) \cdot 10^{-3}$ & $\alpha\left(\mathrm{m}^{2} / \mathrm{s}\right) .10^{7}$ & $\mu_{\mathrm{ap}}(\mathrm{cP})$ \\
\hline 1 & 1,0051 & 1,113 & 105,09 \\
\hline 2 & 1,0014 & 1,118 & 65,56 \\
\hline 3 & 0,9961 & 1,351 & 37,04 \\
\hline 4 & 0,99195 & 1,506 & 34,35 \\
\hline 5 & 0,98635 & 1,862 & 31,3 \\
\hline 6 & 0,99637 & 0,9694 & 135,2 \\
\hline 7 & 0,99197 & 1,121 & 86,17 \\
\hline 8 & 0,98752 & 1,335 & 44,89 \\
\hline 9 & 0,98836 & 1,400 & 45,72 \\
\hline 10 & 0,98705 & 0,8455 & 41,9 \\
\hline 11 & 0,98212 & 1,598 & 40,75 \\
\hline 12 & 0,97432 & 2,087 & 41,48 \\
\hline 13 & 0,98757 & 1,298 & 163,6 \\
\hline 14 & 0,98351 & 1,470 & 140,3 \\
\hline 15 & 0,97798 & 1,516 & 125,17 \\
\hline 16 & 0,97249 & 2,487 & 114,92 \\
\hline 17 & 0,96675 & 4,823 & 109,52 \\
\hline
\end{tabular}

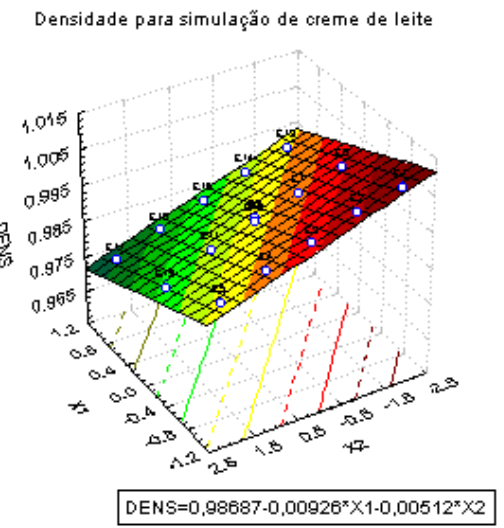

FIGURA 2: Densidade em função de $X_{1}$ e $X_{2}$.

A análise de variância avalia o quanto o modelo de superfície de resposta representa a variação dos dados observados. Para densidade, o modelo representa $99,1 \%$ da variação dos valores observados, a nível de significância de $5 \%$ (Tabela 3). O fator de ajuste para densidade foi de aproximadamente 0,35 , portanto bem acima do valor nominal de 0,05 . Isto significa que a equação tem um ajuste muito bom, com $r^{2}>0,99$. Os limites de confiança dos valores estimados para $B_{0}$, bem como de $\mathrm{B}_{1}$ a $\mathrm{B}_{5}$ estão listados na Tabela 4.

De acordo com a Figura 2, quanto menores os valores de $\mathrm{X}_{1}$ e $\mathrm{X}_{2}$, maior o valor da densidade. Isto é fácil de se visualizar, uma vez que os coeficientes $B_{1}$ e $B_{3}$, os quais estão atrelados à variável $X_{1}$ e $X_{2}$ respectivamente, são negativos (Tabela 4).
TABELA 3. Análise de variância da superfície de resposta, para densidade.

\begin{tabular}{ccccccc}
\hline & SS & GL & MS & $F$ & $p$ & Ajuste do modelo \\
\hline Modelo & 0,00164 & 2 & 0,0008 & 1818 & 0,0006 & \\
Fator de ajuste & $1,18.10^{-5}$ & 12 & $9,79.10^{-7}$ & 2,22 & 0,35 & $99,1 \%$ \\
Erro puro & $8,81.10^{-7}$ & 2 & $4,4.10^{-7}$ & & & \\
Ajuste total & 0,001656 & 16 & & & & \\
\hline
\end{tabular}

${ }^{1} r^{2}=0,99$

TABELA 4. Coeficientes de análise de superfície de resposta para densidade.

\begin{tabular}{cccc}
\hline Coeficiente & $\begin{array}{c}\text { Valor estimado para os } \\
\text { coeficientes }\end{array}$ & Intervalo de confiança de 95\% \\
\hline $\mathrm{B}_{0}$ & 0,98688 & 0,98618 & 0,98757 \\
$\mathrm{~B}_{1}$ & $-0,00926$ & $-0,01016$ & $-0,00836$ \\
$\mathrm{~B}_{2}$ & --- & --- & --- \\
$\mathrm{B}_{3}$ & $-0,00512$ & $-0,00564$ & $-0,00460$ \\
$\mathrm{~B}_{4}$ & --- & --- & --- \\
$\mathrm{B}_{5}$ & --- & --- & --- \\
\hline
\end{tabular}

\section{2 - Difusividade térmica $(\alpha)$}

Os valores de difusividade geral puderam ser calculados através da equação (I), uma vez que o valor da taxa de aquecimento $\mathrm{A}$ geral equivale ao coeficiente angular do gráfico da Figura 3 .

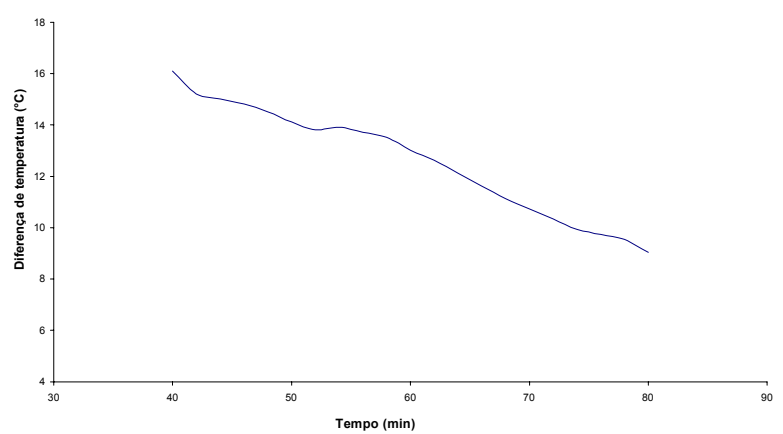

FIGURA 3. Diferença de temperatura versus tempo.

De acordo com a Figura 3, entre 40 e 80 minutos, a curva de difusividade geral versus tempo, para o ensaio 1 , apresentou uma regressão linear onde $r^{2}=0,98$ e inclinação $d T / d t=-0,1698$. O A geral vale 0,7698 . De acordo com a equação (I), calcula-se $\alpha$ a cada temperatura deste ensaio.

A Figura 4 representa o gráfico de superfície de resposta da difusividade térmica em função da porcentagem de gordura e da temperatura.

A resposta encontrada para difusividade térmica representa $63,0 \%$ da variação dos valores observados, a nível de significância de $5 \%$ (Tabela 5 ). 0 fator de ajuste para difusividade térmica foi de aproximadamente 0,23 , portanto bem acima do valor nominal de 0,05 , indicando que o modelo de segunda ordem aplicado 
para difusividade térmica é apropriado para avaliar a variação desta propriedade térmica com a variação de $\mathrm{X}_{1}$ e $\mathrm{X}_{2}$. O modelo de segunda ordem para difusividade térmica (DIF) está apresentado na Figura 4. Os limites de confiança dos valores estimados para $\mathrm{B}_{0}$, bem como de $B_{1}$ a $B_{5}$ estão listados na Tabela 6.

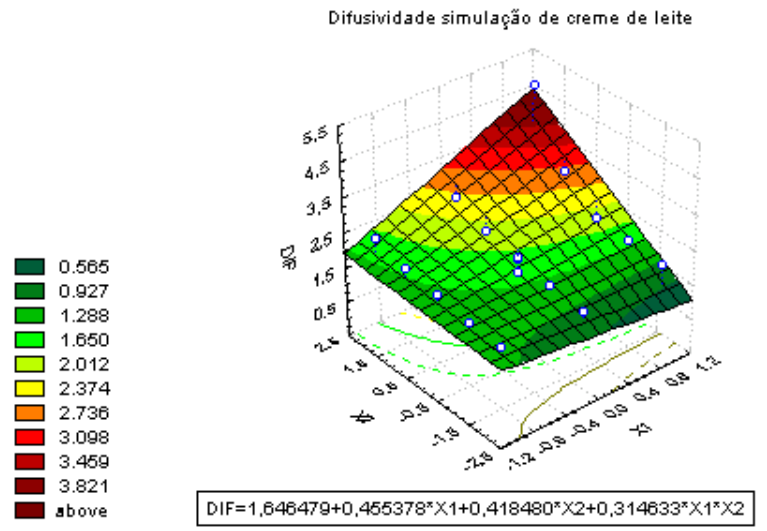

FIGURA 4. Difusividade térmica em função de $X_{1}$ e $X_{2}$.

De acordo com a Figura 4, o valor da difusividade térmica tem uma relação estritamente positiva com as variáveis $X_{1}$ e $X_{2}$, o que significa dizer que quanto maior o valor de $X_{1}$ e $X_{2}$, maior será o valor da difusividade térmica.

TABELA 5. Análise de variância da análise de superfície de resposta para difusividade térmica.

\begin{tabular}{ccccccc}
\hline & SS & GL & MS & F & p & $\begin{array}{c}\text { Ajuste do } \\
\text { modelo }\end{array}$ \\
\hline Modelo & 9,3074 & 3 & 3,1025 & 33,72 & 0,05 & \\
Fator de ajuste & 3,8335 & 11 & 0,3485 & 3,79 & 0,23 & 0,63 \\
Erro puro & 0,1840 & 2 & 0,0920 & & & \\
Ajuste total & 13,3249 & 16 & & & & \\
\hline
\end{tabular}

${ }^{1} r^{2}=0,70$

TABELA 6. Coeficientes de análise de superfície de resposta para difusividade térmica.

\begin{tabular}{cccc}
\hline Coeficiente & $\begin{array}{c}\text { Valor estimado para os } \\
\text { coeficientes }\end{array}$ & \multicolumn{2}{l}{ Intervalo de confiança de 95\% } \\
\hline $\mathrm{B}_{0}$ & 1,6465 & 1,3299 & 1,9629 \\
$\mathrm{~B}_{1}$ & 0,4554 & 0,0427 & 0,8680 \\
$\mathrm{~B}_{2}$ & --- & --- & --- \\
$\mathrm{B}_{3}$ & 0,4185 & 0,1802 & 0,6567 \\
$\mathrm{~B}_{4}$ & --- & --- & --- \\
$\mathrm{B}_{5}$ & 0,3146 & 0,0228 & 0,6064 \\
\hline
\end{tabular}

\section{3 - Viscosidade aparente $\left(\mu_{\mathrm{ap}}\right)$}

De acordo com o procedimento de uso do reômetro Rheotest 2.1, foi necessária a análise da variação da tensão de cisalhamento $\tau$ e da velocidade de deformação $\gamma$ em diferentes rotações desempenhadas pelo reômetro. Com estes valores foi possível determinar os valores do coeficiente de consistência do fluido $(k)$ e do índice de comportamento do fluido (n), e conseqüentemente a viscosidade aparente dos 17 ensaios. Com os dados obtidos pelo Rheotest 2.1, foi possível constatar que a viscosidade aparente, para as 3 formulações, aumenta com o aumento do teor de gordura e diminui com o aumento da velocidade de deformação. Um exemplo da comparação da aparente em relação a estes dois parâmetros, encontra-se na Figura 5. A Tabela 7 mostra um exemplo da relação da viscosidade aparente em função da temperatura e da rotação (ensaio 3).

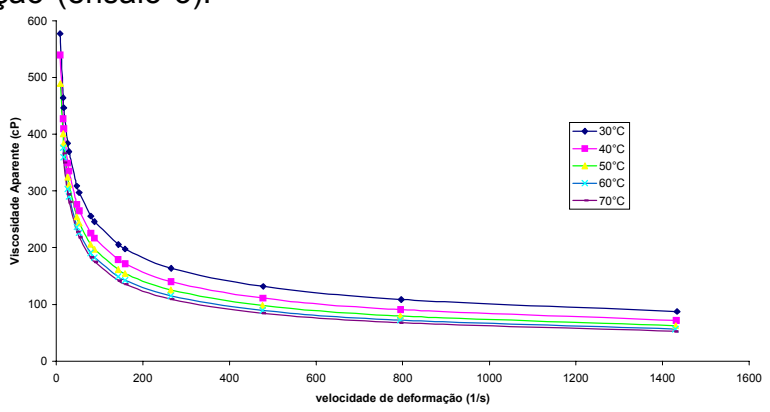

FIGURA 5. Exemplo dos resultados de viscosidade aparente em função da temperatura e da velocidade de deformação (ensaio 3).

TABELA 7. Viscosidade aparente (cP) para o ensaio 3 em funcão da temberatura e da rotacão

\begin{tabular}{cccccc} 
cão da temperatura e da rotacão. \\
Rotação \\
\hline rpm & $30^{\circ} \mathrm{C}$ & $40^{\circ} \mathrm{C}$ & $50^{\circ} \mathrm{C}$ & $60^{\circ} \mathrm{C}$ & $70^{\circ} \mathrm{C}$ \\
1,8 & 577,45 & 539,32 & & & \\
3,24 & 464,36 & 427,35 & 400,74 & 376,08 & 366,56 \\
3,6 & 446,57 & 409,89 & 383,65 & 359,75 & 350,29 \\
5,4 & 384,23 & 349,10 & 324,42 & 303,25 & 294,11 \\
6 & 369,51 & 334,84 & 310,58 & 290,08 & 281,05 \\
9,72 & 308,99 & 276,63 & 254,40 & 236,71 & 228,28 \\
10,8 & 297,15 & 265,32 & 243,56 & 226,43 & 218,14 \\
16,2 & 255,67 & 225,98 & 205,95 & 190,87 & 183,16 \\
18 & 245,87 & 216,74 & 197,17 & 182,58 & 175,02 \\
29,16 & 205,60 & 179,06 & 161,50 & 148,99 & 142,16 \\
32,4 & 197,72 & 171,75 & 154,62 & 142,52 & 135,85 \\
54 & 163,60 & 140,30 & 125,17 & 114,92 & 109,00 \\
97,2 & 131,57 & 111,17 & 98,16 & 89,71 & 84,60 \\
162 & 108,86 & 90,82 & 79,46 & 72,33 & 67,88 \\
291,6 & 87,54 & 71,96 & 62,31 & 56,46 & 52,68 \\
\hline
\end{tabular}

A rotação escolhida para a construção da superfície de resposta foi a de $54 \mathrm{rpm}$, por ser a mais próxima da utilizada em análises de alimentos (60rpm), dentro das limitações do Rheotest. Vale ressaltar que para todas as rotações o ajuste do modelo foi similar, além de 
que o $r^{2}$ foi sempre superior a 0,94.

A resposta encontrada para viscosidade aparente representa $95,2 \%$ da variação dos valores observados, a nível de significância de 1\% (Tabela 8). Os limites de confiança dos valores estimados para $\mathrm{B}_{0}$, bem como de $B_{1}$ a $B_{5}$ estão listados na Tabela 9. Em nenhum caso o fator de ajuste foi significativo, e sempre a interação $\mathrm{X}_{1} \mathrm{X}_{2}\left(\mathrm{~B}_{5}\right)$ foi descartada.

O fator de ajuste para viscosidade aparente foi de aproximadamente 0,035 , portanto acima do valor nominal de 0,01 (Tabela 8). Isto significa que o modelo é plenamente aplicável para se determinar a viscosidade aparente da solução cujos componentes estejam na faixa de estudo deste trabalho. O modelo de segunda ordem para viscosidade aparente (VISC) está apresentado na Figura 6.

TABELA 8. Análise de variância da análise de superfície de

\begin{tabular}{|c|c|c|c|c|c|c|}
\hline \multicolumn{5}{|c|}{ resposta para viscosidade aparente. ${ }_{\mathrm{F}}$} & 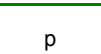 & \multirow{2}{*}{$\begin{array}{l}\text { Ajuste do } \\
\text { modelo }^{1}\end{array}$} \\
\hline Modelo & 29676.55 & 4 & 7419.14 & 1837.83 & $7.10 \mathrm{E}-07$ & \\
\hline Fator de ajuste & 1125.04 & 10 & 112.50 & 27.87 & 0.04 & \\
\hline Erro puro & 8.07 & 2 & 4.04 & & & \\
\hline Ajuste total & 31863.8 & 16 & & & & \\
\hline
\end{tabular}

${ }^{1} r^{2}=0,96$

TABELA 9. Coeficientes de análise de superfície de resposta para viscosidade aparente.

\begin{tabular}{cccc}
\hline Coeficiente & $\begin{array}{c}\text { Valor estimado para os } \\
\text { coeficientes }\end{array}$ & \multicolumn{2}{c}{$\begin{array}{c}\text { Intervalo de confiança de } \\
99 \%\end{array}$} \\
\hline $\mathrm{B}_{0}$ & 51.82 & 43.23 & 60.41 \\
$\mathrm{~B}_{1}$ & 38.02 & 31.71 & 44.32 \\
$\mathrm{~B}_{2}$ & 26.19 & 16.23 & 36.16 \\
$\mathrm{~B}_{3}$ & -18.17 & -21.81 & -14.53 \\
$\mathrm{~B}_{4}$ & 7.34 & 4.45 & 10.22 \\
$\mathrm{~B}_{5}$ & ------- & ------- & ------- \\
\hline
\end{tabular}

De acordo com a Figura 6, o aumento de $\mathrm{X}_{2}$ ocasiona uma diminuição da viscosidade aparente. Isto equivale a dizer que o aumento de temperatura provoca a redução da viscosidade aparente. Por outro lado, o aumento de $X_{1}$ provoca um aumento da viscosidade aparente, ou seja, o aumento do teor de gordura favoreceu o aumento da viscosidade aparente, como era esperado.

\section{4 - CONCLUSÕES}

As equações obtidas apresentaram excelentes resultados, permitindo que, uma vez conhecido o teor de gordura e a temperatura do creme de leite o qual deseja-se saber o valor da densidade, viscosidade aparente ou difusividade térmica, pode-se a princípio simular estas sem a necessidade de medí-las experi- mentalmente, desde que os componentes variem dentro da faixa estudada. Estas equações de modo geral visam otimizar tempo nos processos industriais, como dimensionamento dos equipamentos utilizados no processamento de alimentos.

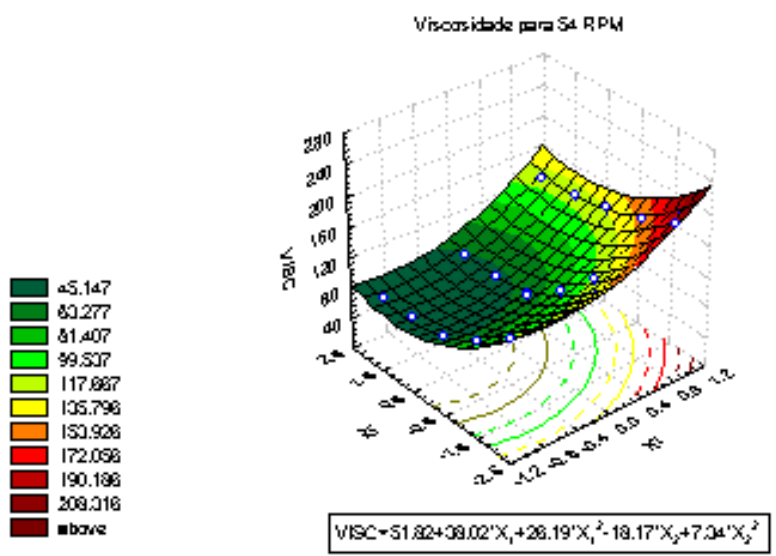

FIGURA 6. Viscosidade aparente em função de $X_{1}$ e $X_{2}$.

\section{5 - REFERÊNCIAS BIBLIOGRÁFICAS}

[1] BARROS, B.; SCARMINIO,I. S.; BRUNS, R. E. 1995. Planejamento e otimização de experimentos. Metodologia de superfície de resposta, Editora da Unicamp. 299p.

[2] BOX, G. E. P.; HUNTER, W.; HUNTER, J. S. 1978. Statistics for experimenters, ed. John Wiley and Sons, New York, NY. 653p.

[3] DICKERSON, R.W. An apparatus for measurements of Thermal Diffusivity of Foods, Food Technology, v. 19, n. 5, p. 198204, 1965.

[4] FERNÁNDEZ-MARTÍN, F.; MONTES F. Thermal conductivity of creams, Journal of Dairy Research, v. 44, p. 103109, 1977

[5] HINRICHS, J. Flow rates for milk and cream in pipelines, Milchwissenschaft, v. 53, n. 3, p. 139-143, 1998.

[6] LIESKE, B.; KONRAD, G. Thermal modification of sodiumcaseinate. 1. Influence of temperature and $\mathrm{pH}$ on selected physical-chemical and functional properties, Milchwissenschaft, v. 49, n. 1, p. 16-20, 1994.

[7] KAILASAPATHY, K.; SINGH, N.; CHERYAN, M. Carrageenanthickened milk base by ultrafiltration, Milchwissenschaft, v. 42, n. 9, p. 491-495, 1997.

[8] MUIR, D. D.; KJAERBYE, H. Quality aspects of UHT cream, Bulletin of the International Dairy Federation, n.315, p. 25-34, 1996

[9] OKOS, M. R.; CHOI, Y., Thermal properties of liquid foods review. In: OKOS, M. R., Physical and Chemical Properties of Foods, Michigan, NY: American Society of Agricultural Engineerers, 1986. p. 35-77.

[10] PHIPPS, L. W. The interrelationship of the viscosity, fat content and temperature of cream between $40^{\circ}$ and $80^{\circ} \mathrm{C}$, Journal of Dairy Research v. 36, p. 417-426, 1969.

[11] POLLEY, S.L.; SNYDER, O.P.; KOTNOUR, P. A compilation of Thermal Properties of Foods, Food Technology, v. 34, n. 11, p. 76-94, 1980.

[12] TEPPER, B. J.; KUANG, T. Perception of fat in a milk model system using muitidimensional scaling, Journal of Sensory 
Studies, v. 11, p. 175-190, 1995.

[13] TEPPER, B. J.; SHAFFER, S. E.; SHEARER C. M. Sensory perception on fat in common foods using two scaling methods, Food Quality and Preference, v. 5, p. 245-
251, 1994.

[14] WILLIAMS, S. (ed.) 1990. Official methods of analysis of the Association of Official Analytical Chemists. $14^{\mathrm{a} a}$ ed., Arlington, AOAC Inc., $1141 \mathrm{p}$.

\section{6-AGRADECIMENTOS}

Ao CNPq pela concessão da bolsa de Iniciação Científica - PIBIC. 